\title{
Possible association between common variants of the phenylalanine hydroxylase $(P A H)$ gene and memory performance in healthy adults
}

\author{
Toshiya Teraishi, Daimei Sasayama, Hiroaki Hori, Noriko Yamamoto, Takashi Fujii, Junko Matsuo, Anna Nagashima, \\ Yukiko Kinoshita, Kotaro Hattori, Miho Ota, Sayaka Fujii and Hiroshi Kunugi*
}

\begin{abstract}
Background: Phenylalanine hydroxylase (PAH) is the enzyme that metabolizes phenylalanine, an essential amino acid required for catecholamine synthesis. Rare mutations in PAH are causal to phenylketonuria (PKU), an autosomal recessive disease characterized by neuropsychiatric symptoms including intellectual disability. We examined whether there is an association between common single nucleotide polymorphisms (SNPs) of PAH and memory performance in the Japanese population.

Methods: Subjects were 599 healthy adults (166 males and 433 females; mean age $43.8 \pm 15.5$ years). The Wechsler Memory Scale-Revised (WMS-R) was administered to all participants to assess memory performance. Genotyping was performed for 6 selected tagging SNPs of PAH (rs1722387, rs3817446, rs1718301, rs2037639, rs10860936 and rs11111419).

Results: Analyses of covariance controlling for sex and education years, indicated a significant association between a SNP (rs2037639) and age-corrected verbal memory index of WMS-R (nominal $p=0.0013$ ) which remained significant after correction for multiple testing ( $p=0.0013<0.0017=0.05 / 30$ tests). Individuals with the $G G$ genotype showed a significantly lower mean verbal memory score, compared with those individuals carrying the AA/AG genotype (106.0 \pm 16.0 vs. $111.7 \pm 13.4 ; p=0.00099$ ). A haplotype block containing two markers of rs2037639 and rs 10860936 was associated with verbal memory index (permutation global $p=0.0091$ ).
\end{abstract}

Conclusions: Our findings suggest that common genetic variations in $P A H$ are associated with verbal memory in healthy adults. Unknown functional polymorphisms in PAH or those in other genes nearby might affect memory performance.

Keywords: Genetic polymorphism, Phenylalanine hydroxylase, Verbal memory, Wechsler Memory Scale-Revised (WMS-R), Association study

\section{Background}

Accumulating evidence suggests that genetic factors influence human complex behavior, including neurocognition throughout the life span $[1,2]$. Studies of single genes that cause monogenic diseases with cognitive impairments will advance understanding of genetic and molecular mechanisms underlying individual differences in neurocognitive performance [3]. Phenylketonuria [PKU; McKusick OMIM 261600], an inborn error of phenylalanine metabolism, causes intellectual disability (i.e., developmental disorder with general cognitive disabilities), seizures, microcephaly, motor disorder, skin rashes and other symptoms, including

\footnotetext{
* Correspondence: hkunugi@ncnp.go.jp

Department of Mental Disorder Research, National Institute of Neuroscience, National Center of Neurology and Psychiatry, 4-1-1, Ogawahigashi, Kodaira, Tokyo 187-8502, Japan
}

(c) 2013 Teraishi et al.; licensee BioMed Central Ltd. This is an Open Access article distributed under the terms of the Creative Commons Attribution License (http://creativecommons.org/licenses/by/2.0), which permits unrestricted use, distribution, and reproduction in any medium, provided the original work is properly cited.

psychiatric conditions such as depression [4-6]. About 98\% of PKU is caused by mutations in phenylalanine hydroxylase $(P A H)$ on chromosome 12q. PAH encodes phenylalanine hydroxylase [EC 1.14.16.1] that converts phenylalanine to tyrosine which is a precursor of the neurotransmitter dopamine and other catecholamines [5,6]. More than 500 mutations in $P A H$ were registered as responsible for PKU (http://www.pahdb.mcgill.ca/) [6].

Interestingly, previous studies $[7,8]$ reported that polymorphisms in $P A H$ confer susceptibility to schizophrenia, a disease characterized by pervasive neurocognitive deficiencies including distinct memory impairment [9], as well as with psychiatric symptoms.

Based on these observations, we hypothesized that common genetic variations in $P A H$ might be associated with neurocognitive function, memory performance in 
particular. The aim of the present study was to examine the possible association of single nucleotide polymorphisms (SNPs) of $P A H$ with memory performance in healthy adults. To our knowledge, there has been no report that examined such an association.

\section{Methods \\ Subjects}

Subjects were 599 healthy Japanese adults (166 males and 433 females; mean age 43.8, standard deviation [SD] 15.5 years). Mean education years of the participants were $15.2 \pm 2.7$ years. All subjects were biologically unrelated and were recruited via advertisements of free local magazines in the western part of Tokyo Metropolitan and our website announcement. All subjects underwent structured interview using the Japanese version of the Mini International Neuropsychiatric Interview (M.I.N.I.) [10,11] and additional unstructured interview by a research psychiatrist to confirm no current or past history of any axis I or II disorders in the Diagnostic and Statistical Manual of Mental Disorders, 4th edition (DSM- IV) criteria [12]. Participants were excluded if they had a current or past contact to psychiatric services, or had a history of severe head injury, serious central nervous system or physical disease, regular use of psychotropics, or substance abuse/dependence.

\section{Assessment of memory performance}

The Japanese version of Wechsler Memory ScaleRevised (WMS-R) $[13,14]$ was administered to all participants $[15,16]$. Raw scores of 5 indices, ie, the verbal, visual and general memory, attention/concentration, and delayed recall calculated from 13 subtests of WMS-R were converted to age-corrected standard scores according to the test manual [14]. The study was approved by the ethics committee at the National Center of Neurology and Psychiatry (NCNP), Japan and was performed in accordance with the Declaration of Helsinki. Every subject gave written informed consent after full explanation of the study aim and protocol.

\section{SNPs-selection and genetic analysis}

Preparation of genomic DNA from venous whole blood of subjects was performed according to standard procedures. We selected 6 tagging SNPs (rs1722387, rs3817446, rs1718301, rs2037639, rs10860936 and rs11111419) throughout $P A H$, encompassing 2 kilobase (kb) of sequence upstream and downstream (between chromosome 12 positions 101,754,235 and 101,837,511), using the 'Tagger' application of the program Haploview 4.2 (http:// www.broad.mit.edu/mpg/haploview/) (version 3.0 release R2) [17]. We used the HapMap genotype data of the Han Chinese from Beijing (CHB) and the Japanese from Tokyo (JPT) and an $\mathrm{r}^{2}$ threshold of 0.8 with a minor allele frequency (MAF) threshold of 0.1. Genotyping of the SNPs was performed using the TaqMan allelic discrimination assay (Applied Biosystems, Foster City, CA, USA) with 10 ng human DNA and $1.5 \mu \mathrm{l} 2 \times$ qPCRTM Mastermix Plus Quick Gold Star (EUROGENTIC, Seraing, Belgium). Polymerase chain reaction was performed with an initial cycle at $95^{\circ} \mathrm{C}$ for 10 minutes followed by 50 cycles of $92^{\circ} \mathrm{C}$ for 15 seconds and $60^{\circ} \mathrm{C}$ for 1 minute in GeneAmp PCR System 9700 (Applied Biosystems). The allele-specific fluorescence was discriminated with an ABI PRISM 7900 Sequence Detection Systems (Applied Biosystems). Genotype data were determined blind to the WMS-R scores. Ambiguous genotype data were excluded from the analysis. The call rate of non-missing genotypes was over $97 \%$.

\section{Statistical analysis}

The statistical package for the social sciences (SPSS) version 11.0 (SPSS Japan, Tokyo) was used for the analysis of variance (ANOVA), analysis of covariance (ANCOVA) and $x^{2}$ test. Demographic characteristics between genotype groups was compared by using ANOVA or $\chi^{2}$ test, as appropriate. The ANCOVA was performed to test the effect of genotype on each score of the 5 indices of WMS-R, controlling for sex and education years. Paired comparisons for verbal memory scores of each genotype were carried out with the ANCOVA controlling for sex and education years. Haploview 4.2 was used to check deviation from the HardyWeinberg equilibrium (HWE) for each SNP, and to calculate measures of linkage disequilibrium (LD). Haplotype blocks were constructed using the default block search algorithm of Gabriel et al. [18]. Haplotypes with frequencies below 1\% were not included in the association analysis. The PLINK statistical package version 1.07 (http://pngu.mgh.harvard. edu/ purcell/plink) [19] was used for haplotype analyses with sex and education years, and empirical $p$ values were obtained based on 10,000 permutations. For the power calculation, G*power software version 3.1.5 (http://www.psycho.uni-duesseldorf.de/abteilungen/aap/gpower3/) was used [20]. Statistical tests were two-tailed and $\mathrm{p}$ values $<0.05$ were considered significant. Since, as to the association analyses between 6 SNPs and 5 indices of WMS-R, we conducted 30 comparisons, critical $\mathrm{p}$ value for multiple testing was set at 0.0017 ( $=0.05 / 30$ tests). Continuous variables were described as mean $\pm \mathrm{SD}$.

\section{Results}

There were no significant group differences in age or smoking status between the genotype groups (e.g., rs2037639 AA/AG/GG) (Additional file 1: Table S1). With respect to education years, a nominally significant group difference between the genotype groups was observed for rs2037639 (Additional file 1: Table S1). Therefore in subsequent analyses, sex and education years were controlled for. Characteristics of the 6 tag SNPs are shown in Table 1. No significant deviation from the HWE was found for the 
Table 1 Characteristics of selected 6 tag SNPs of PAH

\begin{tabular}{|c|c|c|c|c|c|c|c|}
\hline SNP & Chromosome location & Location within $P A H$ & HWE $p$ value & Call rate (\%) & SNP alleles & Minor allele & MAF \\
\hline rs1722387 & 101765200 & Intron 8 & 0.60 & 98.2 & $A / G$ & A & 0.143 \\
\hline rs3817446 & 101784949 & Intron 4 & 0.21 & 99.2 & $A / G$ & A & 0.242 \\
\hline rs1718301 & 101795323 & Intron 4 & 0.64 & 97.3 & $A / G$ & A & 0.166 \\
\hline rs2037639 & 101795480 & Intron 3 & 0.26 & 98.8 & $A / G$ & G & 0.341 \\
\hline rs10860936 & 101807082 & Intron 3 & 0.77 & 98.2 & $\mathrm{C} / \mathrm{T}$ & C & 0.122 \\
\hline rs11111419 & 101809173 & Intron 3 & 0.66 & 98.8 & $\mathrm{~A} / \mathrm{T}$ & $\mathrm{T}$ & 0.223 \\
\hline
\end{tabular}

HWE Hardy-Weinberg Equilibrium, MAF minor allele frequency.

selected 6 markers. Table 2 shows mean scores of the 5 indices of WMS-R and nominal $\mathrm{p}$ values obtained by ANCOVA, in which memory index was a dependent variable, genotypes and sex were independent variables and education years was a covariate. We found 5 out of 30 tests (6 SNPs $\times 5$ indices of WMS-R) were nominally significant without correction for multiple testing. Interestingly, all 5 tests were associations with verbal memory index. Even after Bonferroni correction for multiple testing, the association between rs2037639 and verbal memory index remained significant $(p=0.0013<0.0017=0.05 / 30)$. In addition, we compared indices of WMS-R in only nonsmokers to avoid the possible confounding effects of smoking. ANCOVA controlling for sex and education years showed that the association between rs2037639 and agecorrected verbal memory index of WMS-R was significant for multiple testing $(\mathrm{p}=0.00024<0.0017, \mathrm{~F}=8.5)$. Therefore, we do not think that the association between rs2037639 and age-corrected indices of WMS-R was attributable to smoking status. In subsequent analyses, we focused on the effect of rs2037639 on verbal memory. Figure 1 shows mean verbal memory scores depending on genotypes of rs2037639. There was a significant difference in mean verbal memory score across the three genotype groups $(\mathrm{F}=6.7, \mathrm{df}=2, \mathrm{p}=0.0013)$ (AA genotype: $112.0 \pm$ 12.6, $\mathrm{n}=264$; AG: $111.3 \pm 14.2, \mathrm{n}=254$; GG: $106.0 \pm 16.0$, $\mathrm{n}=75$ ). In paired comparisons, subjects with GG genotype showed a significantly lower mean verbal memory score, compared with subjects with AG $(\mathrm{F}=5.4, \mathrm{df}=1, \mathrm{p}=0.021)$ and AA genotype $(\mathrm{F}=13.4, \mathrm{df}=1, \mathrm{p}=0.00029)$. No significant difference was observed in mean verbal memory score between individuals with AA and AG genotype (data not shown). When the AG and AA genotype groups were combined, ANCOVA controlling for sex and education years showed that the GG genotype group was significantly lower in mean verbal memory score than the AA/AG group $(106.0 \pm 16.0$ vs. $111.7 \pm 13.4 ; F=10.9$, $\mathrm{df}=1, \mathrm{p}=0.00099)$.

Figure 2 represents LD coefficients (D') and constructed two haplotype blocks, block 1 (rs1722387 and rs3817446) and block 2 (rs2037639 and rs10860936), for the 6 SNPs of $P A H$ obtained by the Haploview software. Haplotype analyses for the constructed two haplotype blocks (blocks 1 and 2) using the PLINK software revealed that haplotype block 2, which contained two markers (rs2037639-rs10860936), was significantly associated with verbal memory index (permutation global $\mathrm{p}=0.0091$, Table 3).

Table 2 Association between $P A H$ polymorphisms and indices of the WMS-R

\begin{tabular}{|c|c|c|c|c|c|c|c|c|c|c|c|c|c|c|c|c|c|c|c|}
\hline \multirow{3}{*}{$\begin{array}{c}\begin{array}{c}\text { WMS-R } \\
\text { Index }\end{array} \\
\text { Genotype }\end{array}$} & \multirow{3}{*}{$\begin{array}{l}\text { Mean } \pm \\
\text { S.D. }\end{array}$} & \multicolumn{18}{|c|}{ SNP of $P A H$ gene } \\
\hline & & \multicolumn{3}{|c|}{ rs1722387 } & \multicolumn{3}{|c|}{ rs3817446 } & \multicolumn{3}{|c|}{ rs1718301 } & \multicolumn{3}{|c|}{ rs2037639 } & \multicolumn{3}{|c|}{ rs10860936 } & \multicolumn{3}{|c|}{ rs11111419 } \\
\hline & & $A / A$ & $\mathrm{~A} / \mathrm{G}$ & $\mathrm{G} / \mathrm{G}$ & $\mathrm{A} / \mathrm{A}$ & $\mathrm{A} / \mathrm{G}$ & $\mathrm{G} / \mathrm{G}$ & $\mathrm{A} / \mathrm{A}$ & $\mathrm{A} / \mathrm{G}$ & $\mathrm{G} / \mathrm{G}$ & $\mathrm{A} / \mathrm{A}$ & $A / G$ & $\mathrm{G} / \mathrm{G}$ & $\mathrm{C} / \mathrm{C}$ & $\mathrm{C} / \mathrm{T}$ & $\mathrm{T} / \mathrm{T}$ & $\mathrm{A} / \mathrm{A}$ & $\mathrm{A} / \mathrm{T}$ & $\mathrm{T} / \mathrm{T}$ \\
\hline \multirow{2}{*}{$\begin{array}{l}\text { Verbal } \\
\text { memory }\end{array}$} & \multirow{2}{*}{$\begin{array}{c}110.7 \pm \\
14.0\end{array}$} & 04.6 & 109.6 & 111.2 & 104.8 & 111.9 & 111.0 & 113.5 & 110.1 & 110.9 & 112.0 & 111.3 & 106.0 & 104.8 & 108.9 & 111.4 & 111.6 & 110.5 & 103.9 \\
\hline & & \multicolumn{3}{|c|}{$F=3.57, \mathbf{p}=\mathbf{0 . 0 2 9}$} & \multicolumn{3}{|c|}{$F=3.8, \mathbf{p}=\mathbf{0 . 0 2 3}$} & \multicolumn{3}{|c|}{$F=2.1, p=0.12$} & \multicolumn{3}{|c|}{$F=6.7, \underline{p}=\mathbf{0 . 0 0 1 3}$} & \multicolumn{3}{|c|}{$F=5.5, \mathbf{p}=\mathbf{0 . 0 0 4 2}$} & \multicolumn{3}{|c|}{$F=5.0, p=0.0067$} \\
\hline \multirow{2}{*}{$\begin{array}{c}\text { Visual } \\
\text { memory }\end{array}$} & \multirow{2}{*}{$\begin{array}{c}107.6 \pm \\
11.2\end{array}$} & 106.4 & 107.9 & 107.6 & 107.1 & 108.2 & 107.3 & 110.0 & 108.1 & 107.5 & 107.5 & 108.3 & 107.0 & 107.5 & 107.7 & 107.7 & 107.9 & 107.0 & 109.1 \\
\hline & & \multicolumn{3}{|c|}{$F=0.26, p=0.77$} & \multicolumn{3}{|c|}{$F=0.041, p=0.96$} & \multicolumn{3}{|c|}{$F=0.80, p=0.45$} & \multicolumn{3}{|c|}{$F=0.13, p=0.88$} & \multicolumn{3}{|c|}{$F=0.33, p=0.72$} & \multicolumn{3}{|c|}{$F=1.4, p=0.26$} \\
\hline \multirow{2}{*}{$\begin{array}{l}\text { General } \\
\text { memory }\end{array}$} & \multirow{2}{*}{$\begin{array}{c}111.3 \pm \\
12.8\end{array}$} & 105.8 & 111.3 & 111.5 & 107.0 & 112.7 & 111.2 & 114.5 & 110.9 & 111.5 & 112.0 & 112.1 & 108.1 & 106.2 & 110.8 & 111.7 & 111.9 & 111.2 & 107.4 \\
\hline & & \multicolumn{3}{|c|}{$F=0.89, p=0.41$} & \multicolumn{3}{|c|}{$F=1.9, p=0.15$} & \multicolumn{3}{|c|}{$F=2.4, p=0.089$} & \multicolumn{3}{|c|}{$F=2.8, p=0.060$} & \multicolumn{3}{|c|}{$F=1.5, p=0.22$} & \multicolumn{3}{|c|}{$F=1.4, p=0.26$} \\
\hline Attention/ & & 104.1 & 106.9 & 106.2 & 106.0 & 106.8 & 106.2 & 103.2 & 106.8 & 106.1 & 107.0 & 106.0 & 105.6 & 107.1 & 106.0 & 106.5 & 106.1 & 107.3 & 105.8 \\
\hline & & $F=($ & $7, p=$ & 0.62 & $F=C$ & $.18, p$ & 83 & $\mathrm{~F}=\mathrm{C}$ & $.14, p$ & 87 & $F=$ & $1, p=$ & 35 & $\mathrm{~F}=\mathrm{C}$ & $48, p=$ & 0.62 & $\mathrm{~F}=\mathrm{C}$ & $79, p=$ & 0.45 \\
\hline Delayed & & 105.8 & 111.1 & 110.3 & 107.9 & 111.8 & 110.1 & 112.4 & 109.7 & 110.7 & 110.7 & 111.0 & 108.7 & 109.5 & 110.3 & 110.6 & 111.0 & 110.0 & 109.0 \\
\hline & & $F=$ & $38, p=$ & 0.69 & $\mathrm{~F}=\mathrm{C}$ & $.48, p=$ & & $F=$ & $1.3, p=$ & & $F=($ & $0.44, p=$ & 0.65 & $\mathrm{~F}=\mathrm{C}$ & $87, p=$ & 0.42 & $\mathrm{~F}=\mathrm{C}$ & $.71, p=$ & 0.49 \\
\hline
\end{tabular}

$P$ values of co-dominant model of ANCOVA controlling for education years and sex are shown. $P$ values in boldface and underlined mean nominally significant $(p<0.05)$ and significant for Bonferroni correction for 30 tests $(p<0.0017=0.05 / 30)$, respectively. 


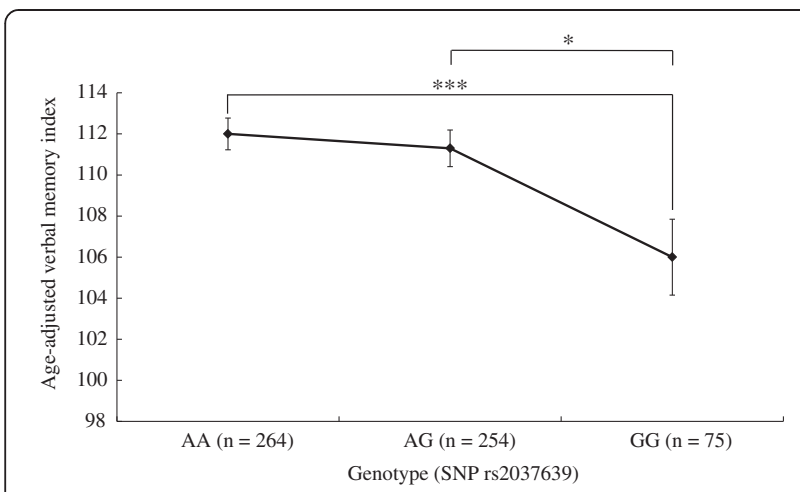

Figure 1 Mean age-adjusted verbal memory scores of WMS-R grouped by genotypes of $P A H$ SNP rs2037639. Data are expressed as mean \pm standard error. ${ }^{*} p<0.05,{ }^{* * *} p<0.001$ : significant differences by analysis of covariance controlling for sex and education years.

\section{Discussion}

We examined whether common genetic variations of $P A H$ are associated with memory performance in Japanese healthy adults. To our knowledge, this is the first attempt to examine such an association. We found a nominally significant association of verbal memory index of WMS-R with 5 SNPs (rs1722387, rs3817446, rs2037639, rs10860936 and rs11111419) of the 6 tag SNPs in PAH. The association between SNP rs2037639 and verbal memory remained significant after correction for multiple testing. Haplotype-based analysis revealed that a haplotype containing two markers (rs2037639-rs10860936) was significantly associated with verbal memory index. These

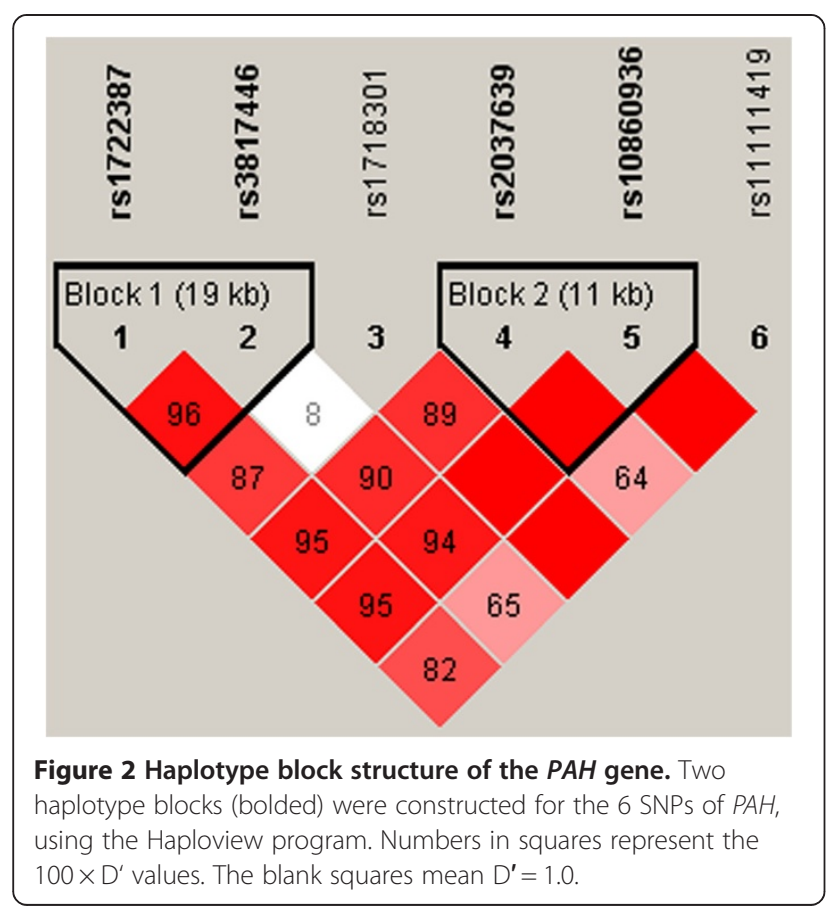

Table 3 Association of PAH haplotypes on verbal memory index of the WMS-R

\begin{tabular}{ccccc}
\hline & Haplotype & Frequency & $\begin{array}{c}\text { Adjusted } \\
\mathbf{p}^{\text {value }}{ }^{\mathbf{a}}\end{array}$ & $\begin{array}{c}\text { Permutation } \\
\text { global } \\
\mathbf{p ~ v a l u e}^{\mathbf{b}}\end{array}$ \\
\hline Block 1 & C-C & 0.753 & 0.060 & \\
(rs1722387- rs3817446) & T-T & 0.140 & 0.12 & 0.13 \\
& C-T & 0.103 & 0.55 & \\
Block 2 & A-T & 0.658 & 0.0068 & \\
(rs2037639-rs10860936) & G-T & 0.220 & 0.25 & 0.0091 \\
& G-C & 0.122 & 0.012 &
\end{tabular}

a Sex- and education years-adjusted $p$ values for haplotypes of each vs. all others. ${ }^{b}$ Empirical $p$ values on the basis of 10,000 permutations.

$P A H$ phenylalanine hydroxylase gene, WMS- $R$ Wechsler Memory Scale-Revised.

results suggest that common functional variants in $P A H$ impact verbal memory performance in healthy adults. Alternatively, there might be a possibility that functional variants in other genes nearby, which would be in linkage disequilibrium with rs2037639 or the haplotype, may play a role. Since there were no PKU patients in our sample, the obtained result cannot be ascribed to mutations responsible for PKU. Further, considering the carrier frequency for PKU of about $1 / 150$ that corresponds to the PKU incidence among Japanese of $1 / 70000$ to $1 / 120000$ $[21,22]$, the number of individuals heterozygous for a rare mutation responsible for PKU is estimated to be less than 5 in our sample. Therefore, it is unlikely that the obtained results are attributable largely to such rare mutations.

Of note, the SNP rs2037639 was previously reported to be associated with schizophrenia in a male Bulgarian sample (nominal $\mathrm{p}=0.03$ ) [7]. Since verbal memory performance is one of the most disturbed neurocognitive functions in schizophrenia, it is possible that the SNP confer susceptibility to schizophrenia, at least in part, via affecting memory performance, an endophenotype of the disease.

There is evidence that rare PKU mutations in $P A H$ are related with impaired working memory in humans $[23,24]$ and chemically induced (ethylnitrosourea, ENU) mouse model of PKU ( $\mathrm{Pah}^{\mathrm{enu} 2}$ ) [25]. However, to our knowledge, there is no report focusing the relationship between common polymorphisms of $P A H$ and cognition. Since there is no direct evidence of functional effects of the SNPs, the mechanism underlying the relationship is currently unclear. The SNP (rs2037639) may have some functional effects such as regulation of transcription of $P A H$. Alternatively, the SNP might be in linkage disequilibrium with an unknown functional polymorphism. The functional effect could alter phenylalanine level, which then alters tyrosine level and activation of tyrosine hydroxylase. Finally the $P A H$ genotypes could affect dopamine level in the brain [26,27], which is related with memory [28]. In addition, neurotransmitter serotonin was also reportedly found to be decreased in PKU [29-31], possibly 
being accounted for by inhibitory effect of phenylalanine on the activity of tryptophan hydroxylase [27]. Serotonin also affects dopamine metabolism and serotonin itself is related with human neurocognition.

Previous genetic studies have shown an association of neurocognition with genes encoding enzymes regulating catecholamine metabolism, such as catechol-Omethyltransferase (COMT) and monoamine oxidase (MAO). Krach et al. reported that the functional COMT Val158Met polymorphism links neural activation pattern during episodic memory tasks [32]. In their study, 84 healthy subjects performed a memory encoding and a retrieval task while they underwent fMRI scanning. Bilateral insula and anterior hippocampus activations were increased linearly with the number of Met alleles (Val/ Val [low PFC dopamine] $<$ Val $/$ Met $<$ Met/Met [high PFC dopamine]) during memory encoding. Giakoumaki et al. found that tolcapone, the COMT inhibitor, enhanced working memory assessed by the letter-number sequencing tasks in healthy subjects with the Val/Val genotype [33]. On the other hand, Enge et al. found that the $M A O A$ variable number of tandem repeats (VNTR) polymorphism, which is located in its promoter region and controls the transcriptional activity of the gene, was associated with working memory-related performance, indicating that $n$-back performance measured by reaction time was poorer in individuals with $M A O-L$ (the short allelic variant and lower enzymatic activity) than in individuals with $M A O-H$ (the long allelic variant and higher enzymatic activity) [34]. Since PAH converts phenylalanine to tyrosine which is a precursor of dopamine and other catecholamines, functional $P A H$ variants could affect synthesis of catecholamines.

There are limitations in the study. The present sample size may have been small, which is subject to type II errors. When we performed a post-hoc power analysis to evaluate the statistical power for the association analysis between verbal memory index and the $P A H$ rs2037639 genotypes, the current sample size had a $80 \%$ power $(\beta=0.2)$ to detect mean difference of 3.3, 4.9, and 5.4 between AA vs. AG, AA vs. GG, and AG vs. GG, respectively, at the $\alpha$ (twotailed) $=5 \%$ level. Further studies with a larger sample size might be required to detect smaller differences. The mechanism by which polymorphisms of $P A H$ influences memory performance is currently unclear, i.e., there is no direct evidence of functional effects of the examined SNPs. Future animal and human studies are therefore warranted to elucidate the mechanism.

\section{Conclusions}

We performed an association study between genetic variations of $P A H$ and memory performance assessed with WMS-R in Japanese healthy adults. We obtained evidence suggesting that genetic variation of $P A H$ (SNP rs2037639 and the haplotype containing two markers, rs2037639 and rs10860936) are associated with verbal memory. Considering the carrier frequency for rare mutations responsible for PKU, our data suggest that common functional polymorphisms in $P A H$ impact verbal memory. Alternatively, functional polymorphisms in other genes nearby may play a role in genetic basis for verbal memory.

\section{Additional file}

Additional file 1: Table S1. Demographic characteristics.

\section{Abbreviations}

ANOVA: Analysis of variance; ANCOVA: Analysis of covariance; COMT: Catechol-O-methyltransferase; DSM- IV: Diagnostic and statistical manual of mental disorders, 4th edition; HWE: Hardy-Weinberg equilibrium; LD: Linkage disequilibrium; MAF: Minor allele frequency; MAO: Monoamine oxidase; MINI: Mini international neuropsychiatric interview; PAH: Phenylalanine hydroxylase; PKU: Phenylketonuria; SNP: Single nucleotide polymorphism; WMS-R: Wechsler memory scale-revised.

\section{Competing interests}

The authors declare that they have no competing interests.

\section{Authors' contributions}

$\Pi \mathrm{T}$ and $\mathrm{HK}$ designed the study and $\Pi \mathrm{\text {wrote }}$ the draft of the manuscript. $\Pi$, DS, HH, KH, MO and SF performed psychiatric assessments for the participants. JM, AN and YK administered the WMS-R. TT and NY performed the genotyping. TF supervised the writing of the paper. HK supervised the project and gave critical comments on the manuscript. All authors contributed to and have approved the final manuscript.

\section{Acknowledgements}

We would like to thank Yumiko Kawamoto, Ikki Ishida, Hidehiko Takeda, Moeko Hiraishi, Ryosuke liboshi, Masayo Ogawa, Yuko Ogawa, Yuuki Yokota and Takahiro Tomizawa for assistance with the neuropsychological tests and all of the volunteers for their participation.

Received: 29 March 2013 Accepted: 25 July 2013

Published: 30 July 2013

\section{References}

1. van Bokhoven $\mathrm{H}$ : Genetic and epigenetic networks in intellectual disabilities. Annu Rev Genet 2011, 45:81-104.

2. McClearn GE, Johansson B, Berg S, Pedersen NL, Ahern F, Petrill SA, Plomin $R$ : Substantial genetic influence on cognitive abilities in twins 80 or more years old. Science 1997, 276(5318):1560-1563.

3. Berger-Sweeney J: Cognitive deficits in Rett syndrome: what we know and what we need to know to treat them. Neurobiol Learn Mem 2011, 96(4):637-646.

4. Surtees R, Blau N: The neurochemistry of phenylketonuria. Eur J Pediatr 2000, 159(Suppl 2):S109-S113.

5. Erlandsen $\mathrm{H}$, Stevens RC: The structural basis of phenylketonuria. $\mathrm{Mol}$ Genet Metab 1999, 68(2):103-125.

6. Scriver CR: The PAH gene, phenylketonuria, and a paradigm shift. Hum Mutat 2007, 28(9):831-845.

7. Talkowski ME, McClain L, Allen T, Bradford LD, Calkins M, Edwards N, Georgieva L, Go R, Gur R, Kirov G, et al: Convergent patterns of association between phenylalanine hydroxylase variants and schizophrenia in four independent samples. Am J Med Genet B Neuropsychiatr Genet 2009, 150B(4):560-569.

8. Richardson MA, Guttler F, Guldberg P, Reilly M, Suckow R, Read L, Clelland J, Chao $\mathrm{H}$, Clelland J: Phenylalanine hydroxylase gene mutation associated with schizophrenia and African-American ethnic status. Schizophr Res 1999, 36:95. 
9. Saykin AJ, Gur RC, Gur RE, Mozley PD, Mozley LH, Resnick SM, Kester DB, Stafiniak P: Neuropsychological function in schizophrenia. Selective impairment in memory and learning. Arch Gen Psychiatry 1991, 48(7):618-624.

10. Sheehan DV, Lecrubier $Y$, Sheehan $\mathrm{KH}$, Amorim $\mathrm{P}$, Janavs J, Weiller $\mathrm{E}$ Hergueta T, Baker R, Dunbar GC: The Mini-International Neuropsychiatric Interview (MINI): the development and validation of a structured diagnostic psychiatric interview for DSM-IV and ICD-10. J Clin Psychiatry 1998, 59(20):22-33-34-57.

11. Otsubo T, Tanaka K, Koda R, Shinoda J, Sano N, Tanaka S, Aoyama H, Mimura M, Kamijima K: Reliability and validity of Japanese version of the Mini-International Neuropsychiatric Interview. Psychiatry Clin Neurosci 2005, 59(5):517-526.

12. American Psychiatric Association: DSM-IV: Diagnostic and Statistical Manual of Mental Disorders. 4. Washington D.C: American Psychiatric Press; 1994.

13. Wechsler D: Wechsler Memory Scale-Revised. San Antonio: Psychological Corp; 1981.

14. Sugishita M: Japanese Wechsler Memory Scale-Revised. Tokyo: Nihonbunkakagakusha; 2001.

15. Hori H, Noguchi H, Hashimoto R, Nakabayashi T, Omori M, Takahashi S, Tsukue R, Anami K, Hirabayashi N, Harada S, et al: Antipsychotic medication and cognitive function in schizophrenia. Schizophr Res 2006, 86(1-3):138-146.

16. Hori H, Yamamoto N, Fujii T, Teraishi T, Sasayama D, Matsuo J, Kawamoto Y, Kinoshita $Y$, Ota M, Hattori K, et al: Effects of the CACNA1C risk allele on neurocognition in patients with schizophrenia and healthy individuals. Sci Rep 2012, 2:634.

17. Barrett JC, Fry B, Maller J, Daly MJ: Haploview: analysis and visualization of LD and haplotype maps. Bioinformatics 2005, 21(2):263-265.

18. Gabriel SB, Schaffner SF, Nguyen H, Moore JM, Roy J, Blumenstiel B, Higgins J, DeFelice M, Lochner A, Faggart M, et al: The structure of haplotype blocks in the human genome. Science 2002, 296(5576):2225-2229.

19. Purcell $S$, Neale B, Todd-Brown $K$, Thomas L, Ferreira MA, Bender D, Maller J, Sklar P, de Bakker PI, Daly MJ, et al: PLINK: a tool set for whole-genome association and population-based linkage analyses. Am J Hum Genet 2007, 81(3):559-575.

20. Faul F, Erdfelder E, Lang AG, Buchner A: G*Power 3: a flexible statistical power analysis program for the social, behavioral, and biomedical sciences. Behav Res Methods 2007, 39(2):175-191.

21. Aoki K, Wada Y: Outcome of the patients detected by newborn screening in Japan. Acta Paediatr Jpn 1988, 30(4):429-434.

22. Wada $Y$, Nakajima H, Irie M, Hirayama M, Suwa S: Newborn mass screening in Japan-1984. Jinrui Idengaku Zasshi 1984, 29(3):277-286.

23. White DA, Nortz MJ, Mandernach T, Huntington K, Steiner RD: Age-related working memory impairments in children with prefrontal dysfunction associated with phenylketonuria. J Int Neuropsychol Soc 2002, 8(1):1-11.

24. Channon S, German E, Cassina C, Lee P: Executive functioning, memory, and learning in phenylketonuria. Neuropsychology 2004, 18(4):613-620.

25. Zagreda L, Goodman J, Druin DP, McDonald D, Diamond A: Cognitive deficits in a genetic mouse model of the most common biochemical cause of human mental retardation. J Neurosci 1999, 19(14):6175-6182.

26. Joseph B, Dyer CA: Relationship between myelin production and dopamine synthesis in the PKU mouse brain. J Neurochem 2003, 86(3):615-626.

27. Ogawa S, Ichinose H: Effect of metals and phenylalanine on the activity of human tryptophan hydroxylase-2: comparison with that on tyrosine hydroxylase activity. Neurosci Lett 2006, 401(3):261-265.

28. Chowdhury R, Guitart-Masip M, Bunzeck N, Dolan RJ, Düzel E: Dopamine modulates episodic memory persistence in old age. J Neurosci 2012, 32(41):14193-14204.

29. McKean CM: The effects of high phenylalanine concentrations on serotonin and catecholamine metabolism in the human brain. Brain Res 1972, 47(2):469-476.

30. Burlina AB, Bonafé L, Ferrari V, Suppiej A, Zacchello F, Burlina AP: Measurement of neurotransmitter metabolites in the cerebrospinal fluid of phenylketonuric patients under dietary treatment. J Inherit Metab Dis 2000, 23(4):313-316.

31. de Groot MJ, Hoeksma M, Blau N, Reijngoud DJ, van Spronsen FJ: Pathogenesis of cognitive dysfunction in phenylketonuria: review of hypotheses. Mol Genet Metab 2010, 99(Suppl 1):S86-S89.

32. Krach S, Jansen A, Krug A, Markov V, Thimm M, Sheldrick AJ, Eggermann T, Zerres K, Stöcker T, Shah NJ, et al: COMT genotype and its role on hippocampal-prefrontal regions in declarative memory. Neurolmage 2010, 53(3):978-984.
33. Giakoumaki SG, Roussos P, Bitsios P: Improvement of prepulse inhibition and executive function by the COMT inhibitor tolcapone depends on COMT Val158Met polymorphism. Neuropsychopharmacology 2008, 33 (13):3058-3068.

34. Enge S, Fleischhauer M, Lesch KP, Reif A, Strobel A: Serotonergic modulation in executive functioning: linking genetic variations to working memory performance. Neuropsychologia 2011, 49(13):3776-3785.

doi:10.1186/1744-9081-9-30

Cite this article as: Teraishi et al:: Possible association between common variants of the phenylalanine hydroxylase $(P A H)$ gene and memory performance in healthy adults. Behavioral and Brain Functions 2013 9:30.

\section{Submit your next manuscript to BioMed Central and take full advantage of:}

- Convenient online submission

- Thorough peer review

- No space constraints or color figure charges

- Immediate publication on acceptance

- Inclusion in PubMed, CAS, Scopus and Google Scholar

- Research which is freely available for redistribution

Submit your manuscript at www.biomedcentral.com/submit
C Biomed Central 